\title{
Interleukin-24 inhibits cell migration and invasion in the neuroblastoma cell line SH-SY5Y
}

\author{
BAOBIAO ZHUO ${ }^{1}$, RONG WANG $^{2}$, HONGWEI ZHANG $^{1}$, HAIHUI QIN $^{1}$, YIYU YIN $^{1}$ and YINGCHUN SHI $^{1}$ \\ ${ }^{1}$ Department of Surgery, Xuzhou Children's Hospital, Xuzhou, Jiangsu 221006; ${ }^{2}$ Department of Ultrasound, \\ The Affiliated Hospital of Xuzhou Medical College, Xuzhou, Jiangsu 221002, P.R. China
}

Received June 29, 2013; Accepted September 5, 2013

DOI: 10.3892/or.2013.2756

\begin{abstract}
Neuroblastomas are common pediatric solid tumors with a variable clinical course; approximately $50 \%$ of patients present with metastatic disease at diagnosis. The development of metastatic lesions often causes a fatal outcome. Therefore, the prevention of metastases during the early stage of tumor development is critical for the improvement of the prognosis of neuroblastoma patients. We previously observed the suppression of neuroblastoma growth in response to overexpression of interleukin-24 (IL-24) in vitro and in vivo. IL-24 exerts its tumor-suppressive effects by multiple mechanisms, including the balance of Bcl-2 family proteins toward the pro-apoptotic pathway and the activation of the caspase cascade. In this study, we used adenovirus-mediated IL-24 (Ad-IL24) to examine the effect of the ectopic production of IL-24 on cell migration and invasion in human neuroblastoma cells. We found that IL-24 effectively inhibits SH-SY5Y neuroblastoma cell migration and invasion by changing subcellular localization and cellular levels of $\beta$-catenin and regulating the levels of proteins associated with cell migration and invasion. Thus, IL-24 should be considered a therapeutic agent that can inhibit primary neuroblastoma growth and that may prevent metastasis.
\end{abstract}

\section{Introduction}

Neuroblastoma is a neuroendocrine tumor, arising from any neural crest element of the sympathetic nervous system (1). Neuroblastoma disease is very heterogeneous and consists of high and low risk disease (2). Low-risk disease, infants aged 18 months and younger with favorable disease characteristics, which is common with observation only or surgery and have a high likelihood of long-term, has disease-free survival. Treating high-risk patients, children aged 18 months and older with stage 4 neuroblastoma and unfavorable disease characteristics, is a bigger challenge due to the presence of widespread

Correspondence to: Professor Yingchun Shi or Professor Yiyu Yin, Department of Surgery, Xuzhou Children's Hospital, 18 Suti North Road, Xuzhou, Jiangsu 221006, P.R. China

E-mail: yingchunshi2000@163.com

E-mail: yiyuyin2000@163.com

Key words: neuroblastoma, interleukin-24, gene therapy, invasion metastatic disease at presentation and a high degree of relapse despite multi-modality treatment $(3,4)$.

In neuroblastoma, $\sim 50 \%$ of patients have metastatic disease at diagnosis, thus creating major challenges for treatment and cure $(5,6)$. Moreover, high-risk patients of neuroblastoma often metastasize and relapse despite initial response to therapies. Frequently, recurrent and metastatic tumors acquire drug resistance or aggressive phenotypes through the selection of rare resistant clones from heterogeneous tumor environment, which can result in major clinical obstacles in the treatment of neuroblastoma (2). Thus, it is imperative to identify novel therapeutic measures to enhance the therapeutic effect and improve the survival of patients with metastatic and recurrent high-risk neuroblastoma disease.

IL-24 is also known as melanoma differentiation-associated 7 (mda-7) due to its first discovery from human melanoma cells by combined treatment with IFN- $\beta$ and MEZ (7). Studies have shown that the overproduction of IL-24 selectively inhibited cancer cell growth of diverse origins by inducing apoptosis with minimal toxicity to normal cells both in vitro and in vivo (8-14). This broad-spectrum antitumor activity of IL-24 is distinct from that of other extensively studied tumor-suppressor genes, and its growth-inhibition properties are independent of the status of p53, pRb, p21 or additional tumor-suppressor genes in cancer cells (15-17).

We previously found the suppression of neuroblastoma growth in response to overexpression of IL-24 in vitro and in vivo. IL-24 exerts its tumor-suppressive effects by multiple mechanisms, including the balance of Bcl-2 family proteins toward the pro-apoptotic pathway and the activation of the caspase cascade (18). Ramesh et al (19) showed that IL-24 inhibited the migration and invasion of human lung cancer cells in vitro. A phase I clinical trial was conducted by Fisher et al (20), who showed IL-24 was safe and promoted significant clinical activity, particularly in the context of patients with metastatic melanoma. We therefore investigated the effects of the IL-24 on migration and invasion in neuroblastoma cells in vitro and attempted to identify the underlying mechanisms of metastasis suppression.

\section{Materials and methods}

Cell culture. As previously described, the human neuroblastoma cell line SH-SY5Y was purchased from Shanghai Cell Bank 
of the Chinese Academic of Sciences (Shanghai, China) (18). The SH-SY5Y cells were grown in EMEM and Ham's F12 (1:1 mixture) $+10 \%$ fetal bovine serum (FBS). Human embryo kidney 293 cells were purchased from Canada Microbix Biosystems Ltd. (Mississauga, Canada). The HEK293 cells were cultured in DMEM medium $+10 \%$ FBS. All cells were maintained in a humidified $37^{\circ} \mathrm{C}$ incubator with $5 \% \mathrm{CO}_{2}$.

Virus production. The construction of replication-defective adenovirus 5 (Ad5) encoding IL-24 gene (Ad-IL24) was previously described (18). The Ad-IL24 and Ad5 carrying reporter gene Green Fluorescent Protein (Ad-GFP) were amplified in HEK 293 cells, purified by cesium chloride centrifugation, and stored at $-80^{\circ} \mathrm{C}$ prior to use.

Cell migration assay. The SH-SY5Y cells were seeded at a density of $6 \times 10^{5}$ cells/well in 6-well tissue culture plates. The next day, cells were infected with Ad-IL24 and Ad-GFP [multiplicity of infection (MOI)=10]. At $6 \mathrm{~h}$ after infection, the cells were trypsinized, washed in PBS and resuspended in serum-free RPMI-1640 medium. A cell migration assay was performed in a 24-well Transwell unit (cat no. CLS3398; Sigma-Aldrich, St. Louis, MO, USA). The lower chambers of the Transwell units were filled with serum-free medium, and the upper chambers were seeded with $1 \times 10^{4}$ cells from each treatment group in triplicate wells. After 24- and 48-h incubations, the cells that had passed through the filter into the lower wells were counted, and the number was expressed as a percentage of the sum of the cells in the upper and lower wells. The experiments were performed 5 times and the results were recorded as the means of these experiments.

In a parallel set of experiments, tumor cells subjected to various treatments as described above were subjected to cell viability assays at 24 and 48 h by MTT, as previously described (18). These experiments were performed to exclude the possibility that the inhibition of cell migration by IL-24 was a result of cytotoxicity.

Cell invasion assay. The SH-SY5Y cells were seeded at a density of $6 \times 10^{5}$ cells/well in 6-well tissue culture plates. The next day, cells were infected with Ad-IL24 and Ad-GFP (MOI=10) or treated with $10 \mu \mathrm{M}$ LY294002 (cat no. 9901; Cell Signaling Technology, Inc., Danvers, MA, USA) or $1 \mu \mathrm{g} / \mathrm{ml}$ MMP-II inhibitor (cat no. 203915-59-7; Santa Cruz Biotechnology, Inc., Santa Cruz, CA, USA). After treatment, cultures were replenished with complete medium. At $6 \mathrm{~h}$ after treatment, cells were trypsinized, washed in PBS, and resuspended in serum-free RPMI-1640 medium. A cell invasion assay was performed in a 24-well Transwell unit coated with Matrigel (cat no. 354578; Becton-Dickinson, Franklin Lakes, NJ, USA). The lower chambers of the Matrigel-coated Transwell units were filled with serum-free medium, and the upper chambers were seeded with $1 \times 10^{4}$ cells from each treatment in triplicate wells. After 24- and 48-h incubations, the cells that had passed through the Matrigel-coated filter membrane into the lower well were counted as a measure of invasion. The invading cells were counted for each treatment and expressed as a percentage of the sum of the cells in the upper and lower wells. Experiments were performed at least 4 times and the results were recorded as the mean of these experiments.
Immunofluorescence staining. The SH-SY5Y cells were seeded at a density of $6 \times 10^{5}$ cells/well in 6-well tissue culture plates. The next day, the cells were infected with Ad-IL24 and Ad-GFP (MOI 10) or treated with PBS. Following treatment, cultures were replenished with complete medium. After $24 \mathrm{~h}$ treatment, cells were fixed in $2 \%$ paraformaldehyde for $10 \mathrm{~min}$, and blocked with $0.5 \%$ Tween-20 for $5 \mathrm{~min}$. Thereafter, cells were incubated with a rabbit monoclonal antibody to $\beta$-catenin (cat no. 9582S; Cell Signaling Technology, Inc.) overnight at $4^{\circ} \mathrm{C}$, followed by $1 \mathrm{~h}$ incubation with a Cy3-conjugated goat anti-rabbit IgG (cat no. AP132C; Millipore, Billerica, MA, USA) in the dark. An epifluorescence Leica microscope was used to observe the samples and a digital camera (Q-imaging) was used to capture the images.

Western blotting. Cells were harvested and lysed, and the cleared lysates (30-50 $\mu \mathrm{g} /$ well) were separated on $10 \%$ Tris-glycine polyacrylamide gel electrophoresis (PAGE) gels under standard conditions. Proteins were then transferred to a nitrocellulose membrane and incubated overnight at $4^{\circ} \mathrm{C}$ with the following primary antibodies: anti-IL-24 (cat no. SAB1407085; Sigma-Aldrich), anti-p85 PI3K (cat no. 4257S), anti-pJNK (cat no. 4668S), anti-p38MAPK (cat no. 4631P, ), anti-pFAK (cat no. 8556S; all reagents were from Cell Signaling Technology, Inc.) or anti- $\beta$-actin (cat no. ab8229; Abcam, Cambridge, MA, USA). The membranes were then washed and incubated with alkaline phosphataseconjugated secondary antibodies in Tris-buffered saline Tween-20 (TBST) for $2 \mathrm{~h}$ and developed using the nitro-blue tetrazolium chloride/5-bromo-4-chloro-3'-indolyphosphate p-toluidine salt (NBT/BCIP) color substrate (Promega Corporation, Madison, WI, USA). The density of the bands on the membrane was scanned and analyzed using an image analyzer.

Gelatin zymography analysis. To determine the effect of Ad-IL24 treatment on MMP production, a gelatin zymography assay was performed. The SH-SY5Y cells were grown in low-serum (1\% FBS) medium and seeded at $6 \times 10^{5}$ cells/well in 6-well tissue culture plates and were infected with Ad-IL24 and Ad-GFP (MOI=10). Cells treated with PBS served as a negative control in these experiments. At $6 \mathrm{~h}$ after infection, the culture medium was removed and replaced with fresh medium containing 1\% FBS. At 24 and $48 \mathrm{~h}$ after infection, cell culture supernatants were collected, clarified by centrifugation, and subjected to electrophoresis in sodium dodecyl sulfate (SDS)-PAGE. The gels were then washed and incubated with reaction buffer $[50 \mathrm{mM}$ Tris- $\mathrm{HCl}(\mathrm{pH}$ 7.4), $0.02 \% \mathrm{NaN}_{3}$, and $10 \mathrm{mM} \mathrm{CaCl}_{2}$ ] with constant shaking for $16 \mathrm{~h}$ at $37^{\circ} \mathrm{C}$, stained and destained. The protein concentration in the culture supernatant was measured to confirm that equal amounts were used for the assays. Relative activities of MMP-2 and MMP-9 were quantified and analyzed using an image analyzer.

Statistical analysis. Values are expressed as the means \pm standard deviation (SD). The statistical analysis of the results was performed using a one-way analysis of the variance (ANOVA) or Student's t-test. P-values $<0.05$ were considered to indicate statistically significant differences. 
A

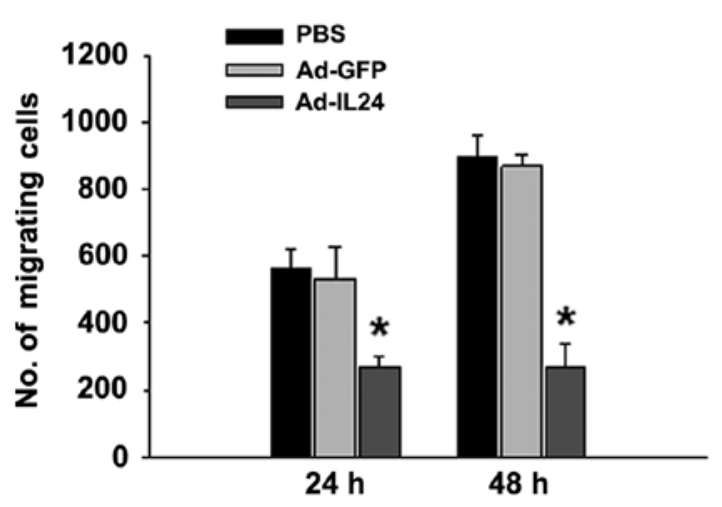

B

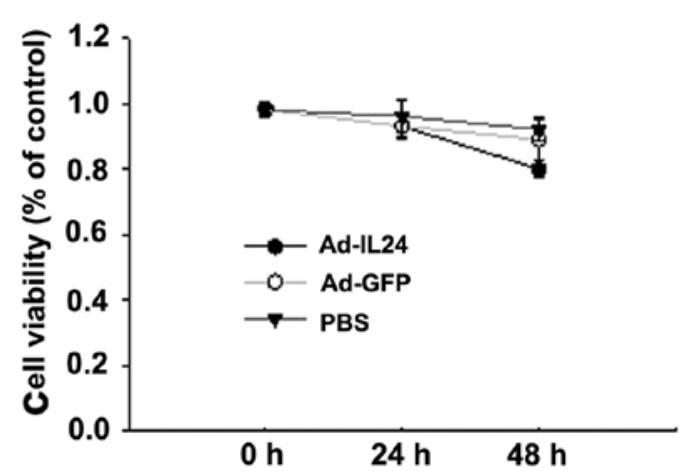

Figure 1. IL-24 inhibits tumor cell migration. The neuroblastoma SH-SY5Y cells were treated with Ad-IL24, Ad-GFP or PBS and analyzed for cell migration and cell viability at 24 and 48 h after treatment. (A) Cells treated with Ad-IL24 were significantly less able to migrate than cells treated with Ad-GFP or PBS. (B) The inhibition of cell migration was not due to cytotoxicity, as determined by detecting the cell viability using MTT assay. No significant inhibition of tumor cell proliferation was observed in Ad-IL24-treated cells compared to Ad-GFP- or PBS- treated cells. Data are means \pm SD from 5 independent experiments $(\mathrm{n}=5) ;{ }^{*} \mathrm{P}<0.05$ vs. Ad-GFP and PBS treatment group. IL-24, interleukin-24; PBS, phosphate-buffered saline.

\section{Results}

IL-24 inhibits neuroblastoma SH-SY5Y cell migration. In the present study we tested the ability of Ad-IL24 to inhibit cell migration in the neuroblastoma cell line SH-SY5Y. Tumor cells treated with Ad-IL24 were significantly less able to migrate than were cells treated with Ad-GFP or PBS (Fig. 1A). The inhibitory effect on cell migration occurred as early as $24 \mathrm{~h}$ in SH-SY5Y cells. To show that the inhibition of cell migration was not due to IL-24-mediated cell death, in a parallel set of experiments, we subjected cells treated with PBS, Ad-GFP and Ad-IL24 to a cell viability assay at 24 and $48 \mathrm{~h}$ after infection. We observed no significant difference in cell viability at 24 and $48 \mathrm{~h}$ after infection, indicating that the inhibition of migration by IL-24 was not due to cell death (Fig. 1B). Note that at $24 \mathrm{~h}$ post-infection, all 3 experimental groups were superimposable, indicating no significant cell death; by $48 \mathrm{~h}$, some cell death occurred; however, with this vector dose, significant Ad-IL24-mediated death was observed only at 72 and $96 \mathrm{~h}$ post-infection; the results are consistent with previous data (18). These results show that IL-24 inhibits tumor cell migration and that the inhibitory effect is not due to cytotoxicity.

IL-24 inhibits neuroblastoma SH-SY5Y cell invasion. SH-SY5Y cells treated with Ad-IL24 were much less invasive, as indicated by the small number of cells on the outer membrane of the Matrigel invasion assay filter, than cells treated with PBS or Ad-GFP (Fig. 2). The number of invading cells was significantly lower after treatment with Ad-IL24 than with PBS or Ad-GFP. We observed the inhibitory effect exerted by IL-24 as early as $24 \mathrm{~h}$ after Ad-IL24 treatment. Furthermore, IL-24-mediated inhibitory effect was similar to the inhibitory effect observed in cells treated with LY294002, a PI3K inhibitor, or with MMP-II inhibitor. A cell viability assay showed that the inhibition was not a result of IL-24mediated cell death. These results show that IL-24 effectively inhibits cell invasion.

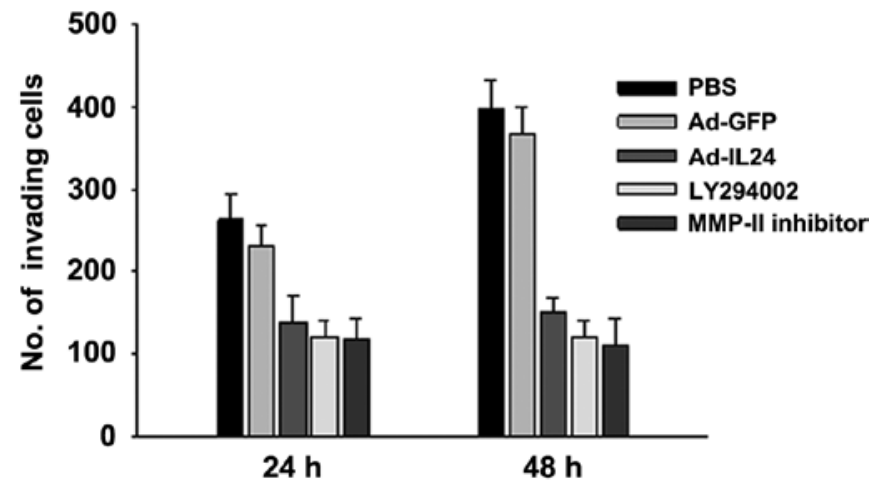

Figure 2. IL-24 inhibits tumor cell invasion. The neuroblastoma SH-SY5Y cells were treated with Ad-IL24, Ad-GFP, PBS, $10 \mu \mathrm{M}$ LY294002 or $1 \mu \mathrm{g} / \mathrm{ml}$ MMP-II inhibitor. After $6 \mathrm{~h}$, cells were harvested, counted and added into the upper wells of Matrigel-coated wells. Cells were allowed to invade by incubation at $37^{\circ} \mathrm{C}$. At 24 and $48 \mathrm{~h}$ after treatment, cells were fixed and stained with crystal violet. Cells that migrated to the lower side of the well were observed and counted under a light microscope at a magnification of $\mathrm{x} 200$. The number of invading cells per treatment was counted in a blind fashion and recorded as the average of 4 separate experiments. Cells treated with Ad-IL24 showed less invasion than cells treated with PBS or Ad-GFP. The inhibitory effect mediated by IL-24 was similar to the inhibitory effect observed with LY294002 and MMP-II inhibitor. IL-24, interleukin-24; PBS, phosphate-buffered saline.

\section{IL-24 affects subcellular localization and upregulates cellular} level of $\beta$-catenin. The subcellular localization of $\beta$-catenin was examined by immunofluorescence. In the PBS- or Ad-GFPtreated cells, positive $\beta$-catenin immunofluorescence staining was observed in the cytoplasm and/or nucleus and was not observed at the plasma membrane (Fig. 3A). In the Ad-IL24treated cells, positive $\beta$-catenin immunofluorescence staining shifted in localization near the plasma membrane and was not observed in the nucleus (Fig. 3A). The cellular level of $\beta$-catenin was examined by western blotting. The intensity of the band corresponding to $\beta$-catenin was stronger in the Ad-IL24-treated cells than in the PBS or Ad-GFP cells (Fig. 3B), indicating that Ad-IL24 increased the cellular level of $\beta$-catenin. 
A

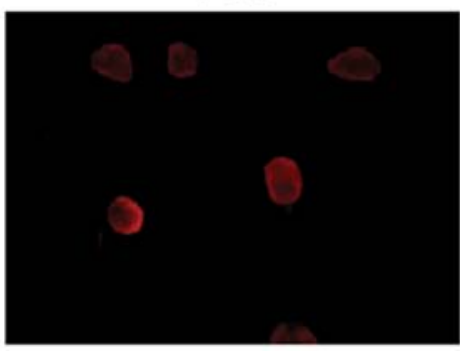

Ad-GFP

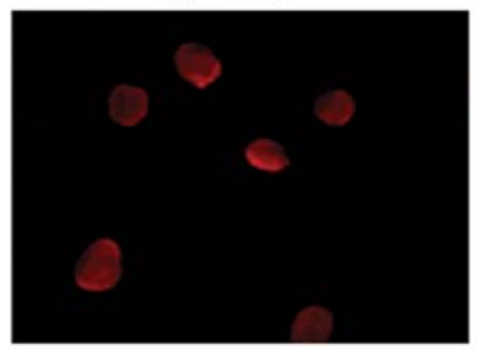

Ad-IL24

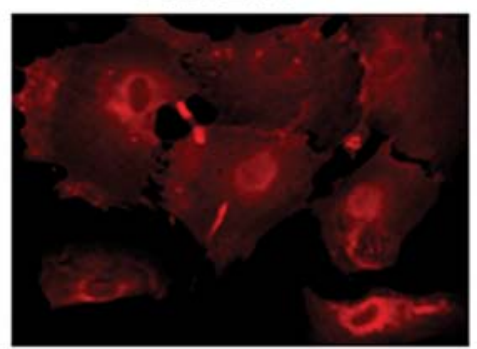

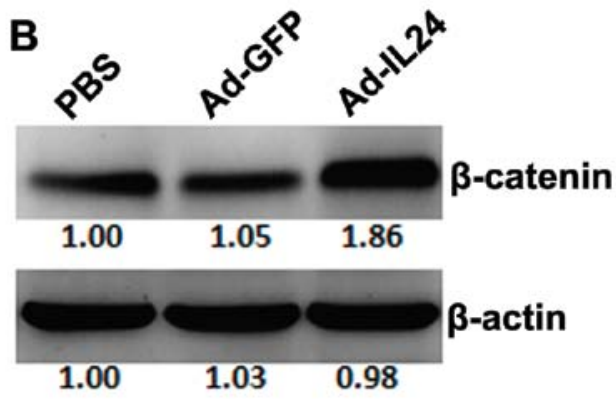

Figure 3. IL-24 changes subcellular localization and upregulates cellular level of $\beta$-catenin. The neuroblastoma SH-SY5Y cells were treated with Ad-IL24, Ad-GFP or PBS and analyzed for cell migration and cell viability at 24 and $48 \mathrm{~h}$ after treatment. (A) Cells were treated for $24 \mathrm{~h}$ and immunofluorescence staining of $\beta$-catenin was performed. In the Ad-IL24-treated cells, positive $\beta$-catenin immunofluorescence staining shifted in localization near the plasma membrane and was not observed in the nucleus. (B) Cells were treated for $24 \mathrm{~h}$ and the expression of $\beta$-catenin and $\beta$-actin was analyzed by western blotting. The intensity of the band corresponding to $\beta$-catenin was stronger in the Ad-IL24-treated cells than in the PBS or Ad-GFP cells. IL-24, interleukin-24; PBS, phosphate-buffered saline.

IL-24 regulates the proteins associated with cell migration and invasion. We next examined the regulation of proteins that are associated with cell migration and invasion signaling pathways by western blot analysis. We did not observe IL-24 production in Ad-GFP-, PBS- or LY294002-treated tumor cells, but high levels of IL-24 production were found in Ad-IL24-treated cells (Fig. 4). Overproduction of the IL-24 protein in tumor cells resulted in decreased production of $\mathrm{p} 85 \mathrm{PI} 3 \mathrm{~K}$ and $\mathrm{pFAK}$ and increased production of $\mathrm{pJNK}$, and p38MAPK compared to PBS- or Ad-GFP-treated cells. We also observed the inhibition of p85 PI3K and pFAK in cells treated with LY294002. The decrease in p85 PI3K expression in cells overproducing IL-24 was greater than that observed with LY294002. Furthermore, LY294002 treatment resulted in increased production of $\mathrm{p} 38 \mathrm{MAPK}$ and $\mathrm{pJNK}$. These results indicate that IL-24, such as LY294002, effectively inhibits PI3K and pFAK while increasing the expression of other signaling proteins.

IL-24 inhibits matrix metalloproteinase production in neuroblastoma SH-SY5Y cells. We next examined tumor cells overproducing IL-24 for MMP regulation by zymography and western blot analysis. Zymography analysis showed that the production of the MMP-2 and MMP-9 proteins was decreased in tumor cells treated with Ad-IL24, compared with cells that were treated with PBS or Ad-GFP (Fig. 5A). The results of zymography analysis correlated with the results of the western blot analysis (Fig. 5B). Thus, Ad-IL24 modulates MMP expression and activity in neuroblastoma SH-SY5Y cells.

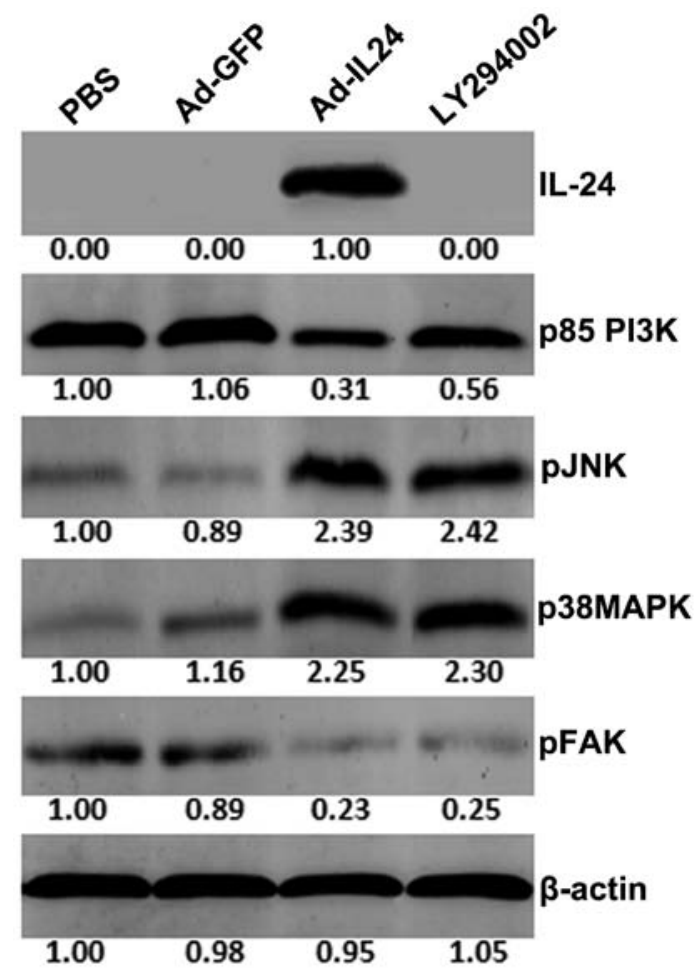

Figure 4. IL-24 downregulates production of proteins associated with migration and invasion. The neuroblastoma SH-SY5Y cells were treated with Ad-IL24, Ad-GFP, PBS or LY294002. Cells were harvested at 48 h, total cell lysates were prepared, and proteins were separated by SDS-PAGE. Proteins were blotted using antibodies against IL-24, p85 PI3K, pJNK, p38MAPK and pFAK. $\beta$-actin was used as a loading control. The relative change in the protein expression was expressed as a ratio with the value for PBS treated cells arbitrarily set to 1 . IL-24, interleukin-24; PBS, phosphate-buffered saline. 
A

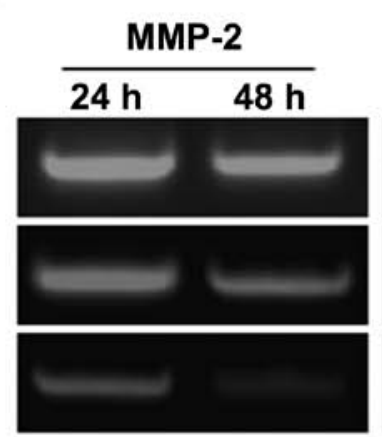

MMP-9

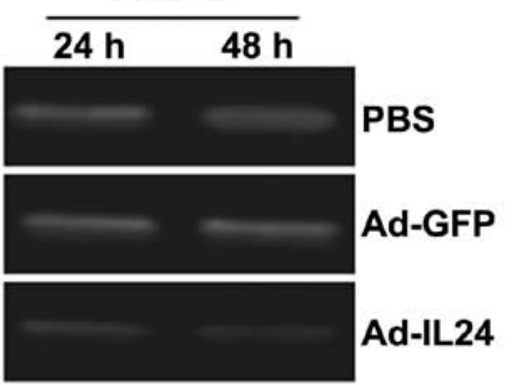

B

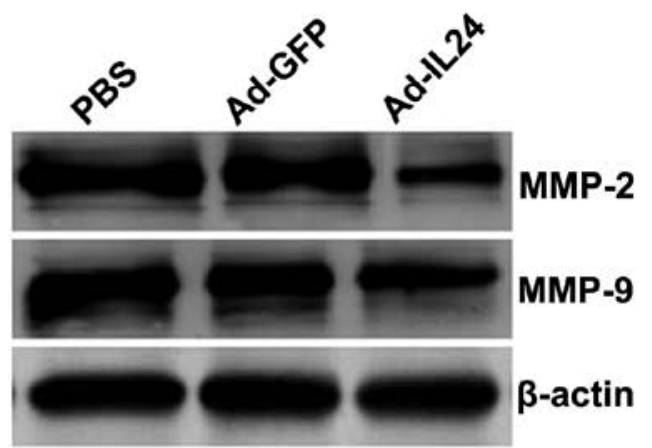

Figure 5. IL-24 inhibits MMP-2 and MMP-9 productions. The neuroblastoma SH-SY5Y cells were treated with Ad-IL24, Ad-GFP or PBS. At 24 and 48 h after treatment, cell supernatant and cell lysate were collected and analyzed for MMP-2 and MMP-9 by zymography and western blot analysis. Inhibition of MMP-2 and MMP-9 was observed in SH-SY5Y cells treated with Ad-IL24. No change in MMP-2 and MMP-9 was observed in cells treated with Ad-GFP or PBS. (A) Inhibition was shown by zymography. (B) Inhibition was shown by western blot analyses. IL-24, interleukin-24; PBS, phosphate-buffered saline.

\section{Discussion}

In the present study, we showed that IL-24 can inhibit the migration and invasion of human neuroblastoma cells. It could be argued that the observed inhibition of cell migration was due to the cytotoxicity of Ad-IL24; however, that was ruled out by a cell proliferation assay. Our results of immunofluorescence showed that IL-24 increases the expression of membranous $\beta$-catenin to enhance cell-cell adhesiveness. Protein production, associated with cell migration and invasion, analysis showed that the inhibition of cell migration and invasion was due to IL-24. The ability of IL-24 to inhibit neuroblastoma cell migration and invasion supports the findings of Ramesh et al (19), who previously reported the inhibition of lung tumor cell migration and invasion by Ad-mda7.

Approximately $50 \%$ of neuroblastoma patients have metastatic disease at diagnosis. The initial step of metastasis is cell detachment from the primary tumor. $\beta$-catenin is essential for cadherin-mediated cell-cell adhesion; the reducing expression of $\beta$-catenin and cadherins at the cell surface is associated with tumor metastasis (21). Loss of membranous $\beta$-catenin occurs commonly in primary colorectal cancer with metastatic potential and in the corresponding colorectal liver metastases (22). Therefore, we examined whether IL-24 affects the subcellular localization and cellular level of $\beta$-catenin in neuroblastoma SH-SY5Y cells. In IL-24-treated cells, immunofluorescence staining of $\beta$-catenin was observed near the plasma membrane. The results of western blotting showed that IL-24-treated cells contained higher levels of $\beta$-catenin than cells that were treated with PBS or Ad-GFP. These findings suggest that IL-24 may promote intracellular adhesion of SH-SY5Y cells. This is the first report to focus on the subcellular localization and cellular level of $\beta$-catenin following IL-24 treatment in tumor cells.

The next step of metastasis is migration of tumor cells to a distant site. Cell migration has previously been shown to be regulated by numerous molecules, including PI3K, p38MAPK, pJNK and FAK (23-27). Inhibition of PI3K and MAPK activity using specific inhibitors impaired epidermal growth factor (EGF)-stimulated cell migration of ovarian tumor cells (28). In our study, we found that IL-24 inhibited cell migration by downregulating the production of the $\mathrm{p} 85$ PI3K and FAK proteins. The inhibitory effect exerted by IL-24 was equivalent to that observed with the PI3K inhibitor LY294002. Furthermore, we examined the regulation of additional signaling molecules p38MAPK and JNK, that have previously been shown to participate in cell migration, and demonstrated increased production of these proteins upon IL-24 expression. Increased expression of these molecules was also observed when cells were treated with LY294002. Thus, IL-24, similar to LY294002, selectively inhibits the PI3K pathway without affecting other signaling pathways, resulting in reduced migration.

In addition to migration, tumor cells need to invade into basement membranes to establish metastasis successfully at a distant site. Tumor cell invasion involves the degradation of the extracellular matrix, and this destruction has been attributed to the activity of proteolytic enzymes (29). MMPs play an important role among the proteases implicated in tumor cells (30). However, the production of MMPs has been observed in many invasive tumor cell lines and during tumor growth (31-33). Evidence that MMPs are involved in invasion and angiogenesis in neuroblastoma comes from the observation that MMP-2 and MMP-9 have been found in several neuroblastoma cell lines and surgical specimens and that the extent of MMP overproduction correlates with prognosis (34-36). In the present study, we found that IL-24-treated cells were less able to migrate and invade, exhibited lower expression and secretion of MMP-2 and MMP-9 compared with PBS- or Ad-GFP-treated cells.

In conclusion, we have shown for the first time that IL-24 inhibits neuroblastoma cell migration and invasion in vitro. Thus, IL-24 gene-based drugs may provide a novel therapeutic strategy for neuroblastoma that can inhibit tumor growth directly via induction of apoptosis and may also prevent tumor invasion and metastasis.

\section{Acknowledgements}

The authors thank Professor Junnian Zheng for providing the plasmid carrying the IL-24 cDNA (Laboratory of Biological Cancer Therapy, Xuzhou Medical College, Xuzhou, China). 


\section{References}

1. Schor NF: Neuroblastoma as a neurobiological disease. J Neurooncol 41: 159-166, 1999.

2. Maris JM: Recent advances in neuroblastoma. N Engl J Med 362: 2202-2211, 2010

3. Gustafson WC and Matthay KK: Progress towards personalized therapeutics: biologic- and risk-directed therapy for neuroblastoma. Expert Rev Neurother 11: 1411-1423, 2011.

4. Isaacs H Jr: Fetal and neonatal neuroblastoma: retrospective review of 271 cases. Fetal Pediatr Pathol 26: 177-184, 2007.

5. Maris JM, Hogarty MD, Bagatell R and Cohn SL: Neuroblastoma. Lancet 369: 2106-2120, 2007.

6. Øra I and Eggert A: Progress in treatment and risk stratification of neuroblastoma: impact on future clinical and basic research. Semin Cancer Biol 21: 217-228, 2011.

7. Jiang H, Lin JJ, Su ZZ, Goldstein NI and Fisher PB: Subtraction hybridization identifies a novel melanoma differentiation associated gene, mda-7, modulated during human melanoma differentiation, growth and progression. Oncogene 11: 2477-2486, 1995.

8. Dent P, Yacoub A, Hamed HA, et al: The development of MDA-7/IL-24 as a cancer therapeutic. Pharmacol Ther 128 375-384, 2010

9. Pataer A, Chada S, Roth JA, Hunt KK and Swisher SG: Development of Ad-mda7/IL-24-resistant lung cancer cell lines. Cancer Biol Ther 7: 103-108, 2008.

10. Zheng M, Bocangel D, Ramesh R, et al: Interleukin-24 overcomes temozolomide resistance and enhances cell death by down-regulation of $O^{6}$-methylguanine-DNA methyltransferase in human melanoma cells. Mol Cancer Ther 7: 3842-3851, 2008.

11. Lebedeva IV, Su ZZ, Vozhilla N, et al: Mechanism of in vitro pancreatic cancer cell growth inhibition by melanoma differentiation-associated gene-7/interleukin-24 and perillyl alcohol. Cancer Res 68: 7439-7447, 2008.

12. Park MA, Yacoub A, Sarkar D, et al: PERK-dependent regulation of MDA-7/IL-24-induced autophagy in primary human glioma cells. Autophagy 4: 513-515, 2008

13. Yan S, Zhang H, Xie Y, et al: Recombinant human interleukin-24 suppresses gastric carcinoma cell growth in vitro and in vivo. Cancer Invest 28: 85-93, 2010.

14. Liu J, Sheng W, Xie Y, et al: The in vitro and in vivo antitumor activity of adenovirus-mediated interleukin-24 expression for laryngocarcinoma. Cancer Biother Radiopharm 25: 29-38, 2010.

15. Jiang H, Su ZZ, Lin JJ, Goldstein NI, Young CS and Fisher PB: The melanoma differentiation associated gene $m d a-7$ suppresses cancer cell growth. Proc Natl Acad Sci USA 93: 9160-9165, 1996.

16. Lebedeva IV, Su ZZ, Chang Y, Kitada S, Reed JC and Fisher PB: The cancer growth suppressing gene $m d a-7$ induces apoptosis selectively in human melanoma cells. Oncogene 21: 708-718, 2002.

17. Su ZZ, Lebedeva IV, Sarkar D, et al: Melanoma differentiation associated gene-7, $m d a-7 /$ IL-24, selectively induces growth suppression, apoptosis and radiosensitization in malignant gliomas in a p53-independent manner. Oncogene 22: 1164-1180, 2003.

18. Zhuo B, Wang R, Yin Y, et al: Adenovirus arming human IL-24 inhibits neuroblastoma cell proliferation in vitro and xenograft tumor growth in vivo. Tumour Biol 34: 2419-2426, 2013.

19. Ramesh R, Ito I, Gopalan B, Saito Y, Mhashilkar AM and Chada S: Ectopic production of MDA-7/IL-24 inhibits invasion and migration of human lung cancer cells. Mol Ther 9: 510-518, 2004.
20. Fisher PB, Sarkar D, Lebedeva IV, et al: Melanoma differentiation associated gene-7/interleukin-24 (mda-7/IL-24) novel gene therapeutic for metastatic melanoma. Toxicol Appl Pharmacol 224: 300-307, 2007.

21. Fujioka T, Takebayashi Y, Kihana T, et al: Expression of E-cadherin and $\beta$-catenin in primary and peritoneal metastatic ovarian carcinoma. Oncol Rep 8: 249-255, 2001.

22. Hugh TJ, Dillon SA, O'Dowd G, et al: $\beta$-catenin expression in primary and metastatic colorectal carcinoma. Int J Cancer 82: 504-511, 1999.

23. Wang F, Nohara K, Olivera A, Thompson EW and Spiegel S: Involvement of focal adhesion kinase in inhibition of motility of human breast cancer cells by sphingosine 1-phosphate. Exp Cell Res 247: 17-28, 1999.

24. Neudauer CL and McCarthy JB: Insulin-like growth factor I-stimulated melanoma cell migration requires phosphoinositide 3-kinase but not extracellular-regulated kinase activation. Exp Cell Res 286: 128-137, 2003.

25. Goncharova EA, Ammit AJ, Irani C, et al: PI3K is required for proliferation and migration of human pulmonary vascular smooth muscle cells. Am J Physiol Lung Cell Mol Physiol 283: L354-L363, 2002.

26. Kadri Z, Petitfrère E, Boudot C, et al: Erythropoietin induction of tissue inhibitors of metalloproteinase- 1 expression and secretion is mediated by mitogen-activated protein kinase and phosphatidylinositol 3-kinase pathways. Cell Growth Differ 11: 573-580, 2000.

27. Lakka SS, Jasti SL, Kyritsis AP, et al: Regulation of MMP-9 (type IV collagenase) production and invasiveness in gliomas by the extracellular signal-regulated kinase and jun amino-terminal kinase signaling cascades. Clin Exp Metastasis 18: 245-252, 2000.

28. Ellerbroek SM, Halbleib JM, Benavidez M, et al: Phosphatidylinositol 3-kinase activity in epidermal growth factor-stimulated matrix metalloproteinase-9 production and cell surface association. Cancer Res 61: 1855-1861, 2001.

29. Goldfarb RH and Liotta LA: Proteolytic enzymes in cancer invasion and metastasis. Semin Thromb Hemost 12: 294-307, 1986.

30. Matrisian LM: The matrix-degrading metalloproteinases. Bioessays 14: 455-463, 1992.

31. Shapiro SD: Matrix metalloproteinase degradation of extracellular matrix: biological consequences. Curr Opin Cell Biol 10: 602-608, 1998

32. Pritchard SC, Nicolson MC, Lloret C, et al: Expression of matrix metalloproteinases 1, 2, 9 and their tissue inhibitors in stage II non-small cell lung cancer: Implications for MMP inhibition therapy. Oncol Rep 8: 421-424, 2001.

33. Nakopoulou L, Tsirmpa I, Alexandrou P, et al: MMP-2 protein in invasive breast cancer and the impact of MMP-2/TIMP-2 phenotype on overall survival. Breast Cancer Res Treat 77: $145-155,2003$.

34. Lu HF, Lai KC, Hsu SC, et al: Involvement of matrix metalloproteinases on the inhibition of cells invasion and migration by emodin in human neuroblastoma SH-SY5Y cells. Neurochem Res 34: 1575-1583, 2009.

35. Ribatti D, Surico G, Vacca A, et al: Angiogenesis extent and expression of matrix metalloproteinase- 2 and -9 correlate with progression in human neuroblastoma. Life Sci 68: 1161-1168, 2001.

36. Ara T, Fukuzawa M, Kusafuka T, et al: Immunohistochemical expression of MMP-2, MMP-9, and TIMP-2 in neuroblastoma: association with tumor progression and clinical outcome. J Pediatr Surg 33: 1272-1278, 1998. 